\title{
Lagrangian cohomological couplings among vector fields and matter fields
}

\author{
C. Bizdadea, E. M. Cioroianu ${ }^{\dagger}$ M. T. Miaută, \\ I. Negru; S. O. Saliu ${ }^{\S}$ \\ Faculty of Physics, University of Craiova \\ 13 A. I. Cuza Str., Craiova RO-1100, Romania
}

November 16, 2018

\begin{abstract}
Consistent couplings between a set of vector fields and a system of matter fields are analysed in the framework of Lagrangian BRST cohomology.
\end{abstract}

PACS number: 11.10.Ef

\section{Introduction}

The antifield-BRST symmetry [1]-[2] viewed from a cohomological perspective provided a helpful instrument for analysing consistent interactions in gauge theories [3]-[6]. In this light, many models of interest in theoretical physics, like, for example, the Yang-Mills theory [7], the Chapline-Manton model [8], $p$-forms and chiral $p$-forms [9]-[13], as well as nonlinear gauge theories [14, have been derived along the deformation of the master equation. Also, it is important to notice the deformation results connected to

\footnotetext{
*e-mail address: bizdadea@central.ucv.ro

$\dagger$ †-mail address: manache@central.ucv.ro

‡e-mail address: inegru@central.ucv.ro

§e-mail address: osaliu@central.ucv.ro
} 
Einstein's gravity theory [15] and four- and eleven-dimensional supergravity [16]. Meanwhile, the problem of obtaining consistent deformations has naturally found its extension at the Hamiltonian level [17]-20].

In this paper we investigate consistent Lagrangian couplings that can be added between a set of vector fields and a system of matter fields (of spin 0 , respectively, 1/2) by means of the deformation of the master equation. This approach represents an extension of our former results exposed in 21 on the abelian case. Our treatment goes as follows. We begin with a "free" action written as the sum between the action for a set of vector fields and an action describing a matter theory, and construct the corresponding "free" Lagrangian BRST differential $s$, which is found to decompose as the sum between the Koszul-Tate differential and the exterior derivative along the gauge orbits, $s=\delta+\gamma$. Further, we analyse the deformation of the associated solution to the master equation. The first-order deformation belongs to $H^{0}(s \mid d)$, where $d=d x^{\mu} \partial_{\mu}$ denotes the exterior space-time derivative. The computation of the cohomological space $H^{0}(s \mid d)$ proceeds by expanding the co-cycles according to the antighost number, and by subsequently using the cohomological spaces $H(\gamma)$ and $H(\delta \mid d)$. The first-order deformation reveals two different types of couplings. One involves only the vector fields (the cubic vertex of Yang-Mills theory), and requires no supplementary assumptions. The other demands that the cohomological space $H_{1}(\delta \mid d)$ is not empty, or, in other words, that the matter theory should display some global invariances that result in some conserved currents $j_{a}^{\mu}$. Consequently, it follows that the second type of couplings from the first-order deformation is written in the form $j_{a}^{\mu} A_{\mu}^{a}$. The consistency of the first-order deformation asks that the deformed gauge algebra is Lie, and, moreover, outputs precisely the well-known quartic vertex of Yang-Mills theory. Concerning the higher-order deformations, there appear two main situations. If the conserved currents $j^{\mu}{ }_{a}$ transform under the gauge version of the rigid symmetries of the matter fields according to the adjoint representation of the Lie gauge algebra, then all the other deformations involving the matter fields, of order two and higher, vanish. In the opposite case, at least the second-order deformation implying matter fields is non-vanishing, but in principle there might be other non-trivial terms.

The paper is structured in five sections. In Sec. 2 we briefly review the problem of deformation of the master equation. Sec. 3 focuses on the analysis of the consistent couplings between a set of vector fields and a collection of matter fields by means of deforming the solution to the master equation. In 
Sec. 4 we apply our treatment to two cases of interest, where the role of the matter fields is played by a set of real scalar fields, respectively, by a collection of Dirac fields. Sec. 5 ends the paper with some conclusions.

\section{Deformation of the master equation: a brief review}

We begin with a "free" gauge theory, described by an action $S_{0}\left[\Phi^{\alpha_{0}}\right]$, invariant under some gauge transformations

$$
\delta_{\epsilon} \Phi^{\alpha_{0}}=Z_{\alpha_{1}}^{\alpha_{0}} \epsilon^{\alpha_{1}}, \frac{\delta S_{0}}{\delta \Phi^{\alpha_{0}}} Z_{\alpha_{1}}^{\alpha_{0}}=0,
$$

and consider the problem of consistent interactions that can be introduced among the fields $\Phi^{\alpha_{0}}$, so the couplings preserve the original number of gauge symmetries. This matter is addressed by means of reformulating the problem of constructing consistent interactions as a deformation problem of the solution to the master equation corresponding to the "free" theory [3]. Such a reformulation is possible due to the fact that the solution to the master equation contains all the information on the gauge structure of the theory. If a consistent interacting gauge theory can be constructed, then the solution $\tilde{S}$ to the master equation associated with the "free" theory can be deformed into a solution $S$,

$$
\begin{aligned}
& \tilde{S} \rightarrow S=\tilde{S}+g S_{1}+g^{2} S_{2}+\cdots= \\
& \tilde{S}+g \int d^{D} x a+g^{2} \int d^{D} x b+\cdots,
\end{aligned}
$$

of the master equation for the deformed theory

$$
(S, S)=0
$$

that displays the same ghost and antifield spectra. According to the deformation parameter $g$, eq. (3) splits into

$$
\begin{gathered}
(\tilde{S}, \tilde{S})=0, \\
2\left(S_{1}, \tilde{S}\right)=0 \\
2\left(S_{2}, \tilde{S}\right)+\left(S_{1}, S_{1}\right)=0,
\end{gathered}
$$




$$
\left(S_{3}, \tilde{S}\right)+\left(S_{1}, S_{2}\right)=0
$$

While eq. (4) is fulfilled by hypothesis, the next one requires that $S_{1}$ is a co-cycle of the "free" BRST differential $s \bullet=(\bullet, \tilde{S})$. However, only cohomologically non-trivial solutions to (5) should be taken into account, as the BRST-exact ones can be eliminated by a (in general non-linear) field redefinition, such that $S_{1}$ pertains to the ghost number zero cohomological space of $s, H^{0}(s)$, which is known to be isomorphic to the space of physical observables of the "free" theory. It has been shown in [3], 22] on behalf of the triviality of the antibracket in the cohomology that there are no obstructions in finding solutions to the remaining eqs. ((6-7), etc.). Unfortunately, the resulting interactions may not be local, and there might appear obstructions if one insists on the locality of the deformations. The analysis of these obstructions can be done with the help of cohomological techniques. However, as it will be seen below (see Sec. 4), the interaction terms to be dealt with turn out to be local.

\section{Couplings among vector fields and matter fields from BRST cohomology}

The scope of this section is to analyse the consistent interactions among vector fields and matter fields with the help of the Lagrangian BRST deformation procedure.

\subsection{Free BRST differential}

We start from a "free" theory whose Lagrangian action is written as the sum between the action for a collection of vector fields $A_{\mu}^{a}$ and an action involving some matter fields $y^{i}$

$$
\tilde{S}_{0}^{L}\left[A_{\mu}^{a}, y^{i}\right]=\int d^{D} x\left(-\frac{1}{4} F_{\mu \nu}^{a} F_{a}^{\mu \nu}+\mathcal{L}_{0}\left(y^{i}, \partial_{\mu} y^{i}, \ldots, \partial_{\mu_{1}} \cdots \partial_{\mu_{k}} y^{i}\right)\right),
$$

where the field strength is defined as $F_{\mu \nu}^{a}=\partial_{\mu} A_{\nu}^{a}-\partial_{\nu} A_{\mu}^{a}$. The Grassmann parity of a matter field $y^{i}$ is denoted by $\varepsilon_{i}$. We assume that the matter fields 
possess no gauge invariances of their own, hence action (8) is invariant under the gauge transformations

$$
\delta_{\epsilon} A_{\mu}^{a}=\partial_{\mu} \epsilon^{a}, \delta_{\epsilon} y^{i}=0 .
$$

Then, the solution to the master equation for this "free" theory reads as

$$
\tilde{S}=\tilde{S}_{0}^{L}\left[A_{\mu}^{a}, y^{i}\right]+\int d^{D} x A_{a}^{* \mu} \partial_{\mu} \eta^{a}
$$

where $\eta^{a}$ are the fermionic ghosts, and the star variables represent the antifields of the corresponding fields/ghosts. The antifields $A_{a}^{* \mu}$ of the vector fields are fermionic, while those of the ghosts, $\eta_{a}^{*}$, are bosonic. The grading of the BRST differential is named ghost number $(\mathrm{gh})$, and is defined like the difference between the degree of the exterior derivative along the gauge orbits, pure ghost number (pgh), and the grading of the Koszul-Tate differential, antighost number (antigh), where

$$
\begin{gathered}
\operatorname{pgh}\left(A_{\mu}^{a}\right)=\operatorname{pgh}\left(y^{i}\right)=\operatorname{pgh}\left(A_{a}^{* \mu}\right)=\operatorname{pgh}\left(y_{i}^{*}\right)=\operatorname{pgh}\left(\eta_{a}^{*}\right)=0, \operatorname{pgh}\left(\eta^{a}\right)=1, \\
\operatorname{antigh}\left(A_{\mu}^{a}\right)=\operatorname{antigh}\left(y^{i}\right)=0, \operatorname{antigh}\left(A_{a}^{* \mu}\right)=\operatorname{antigh}\left(y_{i}^{*}\right)=1, \\
\operatorname{antigh}\left(\eta_{a}^{*}\right)=2, \operatorname{antigh}\left(\eta^{a}\right)=0 .
\end{gathered}
$$

The BRST symmetry of the "free" theory, $s \bullet=(\bullet, \tilde{S})$, simply decomposes as the sum between the Koszul-Tate differential and the exterior longitudinal derivative

$$
s=\delta+\gamma,
$$

where these operators act on the generators of the BRST complex through the relations

$$
\begin{gathered}
\delta A_{\mu}^{a}=0, \delta y^{i}=0, \delta \eta^{a}=0 \\
\delta A_{a}^{* \mu}=-\partial_{\nu} F_{a}^{\nu \mu}, \delta y_{i}^{*}=-\frac{\delta^{L} \mathcal{L}_{0}}{\delta y^{i}}, \delta \eta_{a}^{*}=-\partial_{\mu} A_{a}^{* \mu}, \\
\gamma A_{\mu}^{a}=\partial_{\mu} \eta^{a}, \gamma y^{i}=0, \gamma \eta^{a}=0, \\
\gamma A_{a}^{* \mu}=0, \gamma y_{i}^{*}=0, \gamma \eta_{a}^{*}=0
\end{gathered}
$$

which will be used in the sequel during the deformation process. 


\section{$3.2 \quad$ First-order deformations}

For determining the consistent interactions that can be added to the "free" gauge theory under discussion, we examine the eqs. (5-7), etc., by relying on the action of the "free" BRST differential. Using the notations from (2), the local form of the eq. (5) is

$$
s a=\partial_{\mu} n^{\mu},
$$

for some local $n^{\mu}$, and it holds if and only if $a$ is a $s$-co-cycle modulo $d$. In order to solve this eq., we develop $a$ according to the antighost number,

$$
a=a_{0}+a_{1}+\cdots+a_{j}
$$

where antigh $\left(a_{i}\right)=i$, and the last term in $a$ can be assumed to be annihilated by $\gamma, \gamma a_{j}=0$. Thus, we need the cohomology of $\gamma, H(\gamma)$, in order to determine the components of highest antighost number in $a$. From (17 18) it is simple to see that the cohomology of $\gamma$ is spanned by $F_{\mu \nu}^{a}=\partial_{[\mu} A_{\nu]}^{a}, y^{i}$ and their derivatives, by the antifields and their derivatives, as well as by the undifferentiated ghosts $\eta^{a}$. (The derivatives of the ghosts do not bring any contribution to $H(\gamma)$ as they are $\gamma$-exact.) If we denote by $e^{M}\left(\eta^{a}\right)$ a basis in the space of the polynomials in the ghosts, it follows that the general solution to the equation $\gamma \alpha=0$ (up to a trivial term) takes the form

$$
\alpha=\alpha_{M}\left(\left[F_{\mu \nu}^{a}\right],\left[y^{i}\right],\left[A_{a}^{* \mu}\right],\left[y_{i}^{*}\right],\left[\eta_{a}^{*}\right]\right) e^{M}\left(\eta^{a}\right)
$$

where the notation $f[q]$ signifies that $f$ depends on $q$ and its derivatives up to a finite order. Relying on the fact that $\mathrm{gh}(a)=0$, it results that $\operatorname{pgh}\left(a_{i}\right)=i$, hence from (21) we have that the last representative in $a$ is of the type

$$
a_{j}=\frac{1}{(j+1) !} a_{a_{1} \cdots a_{j}}\left(\left[F_{\mu \nu}^{a}\right],\left[y^{i}\right],\left[A_{a}^{* \mu}\right],\left[y_{i}^{*}\right],\left[\eta_{a}^{*}\right]\right) \eta^{a_{1}} \cdots \eta^{a_{j}}
$$

The eq. (19) projected on antighost number $(j-1)$ becomes

$$
\delta a_{j}+\gamma a_{j-1}=\partial_{\mu} m^{\mu} .
$$

The last equation possesses solutions if all $a_{a_{1} \cdots a_{j}}$ pertain to $H_{j}(\delta \mid d)$. In the meantime, applying the results from [22], it follows that $H_{j}(\delta \mid d)$ vanishes for $j>2$, so we can assume that the first-order deformation stops after the first three elements

$$
a=a_{0}+a_{1}+a_{2},
$$


with $a_{2}=\frac{1}{2} a_{a b} \eta^{a} \eta^{b}$ and $a_{a b}=-a_{b a}$ from $H_{2}(\delta \mid d)$, i.e.

$$
\delta a_{a b}=\partial_{\mu} k_{a b}^{\mu} .
$$

Nevertheless, the most general element from $H_{2}(\delta \mid d)$ can be represented as

$$
\lambda=\lambda^{a} \eta_{a}^{*}
$$

with constant $\lambda^{a}$, such that $\delta \lambda=\partial_{\mu}\left(-\lambda^{a} A_{a}^{* \mu}\right)$. These considerations lead to

$$
a_{a b}=-f_{a b}^{c} \eta_{c}^{*}
$$

with $f_{a b}^{c}=-f_{b a}^{c}$ some constants. Combining the above results, we can state that the antighost number two component of $a$ can be written in the form

$$
a_{2}=-\frac{1}{2} f_{a b}^{c} \eta_{c}^{*} \eta^{a} \eta^{b}
$$

The eq. (19) projected on antighost number one becomes $\delta a_{2}+\gamma a_{1}=\partial_{\mu} k^{\mu}$. Using (28), by direct computation we find

$$
\delta a_{2}=\partial_{\mu}\left(\frac{1}{2} f_{a b}^{c} A_{c}^{* \mu} \eta^{a} \eta^{b}\right)-\gamma\left(f_{a b}^{c} A_{c}^{* \mu} A_{\mu}^{b} \eta^{a}+y_{i}^{*} T_{a}^{i} \eta^{a}\right),
$$

where $T_{a}^{i}$ are some local functions of $y^{i}$ and their derivatives. From (29) we read the antighost number one term of the first-order deformation

$$
a_{1}=f_{a b}^{c} A_{c}^{* \mu} A_{\mu}^{b} \eta^{a}+y_{i}^{*} T^{i}{ }_{a}^{a} \eta^{a} .
$$

In order to solve the eq. controlling the antighost number zero deformation, $\delta a_{1}+\gamma a_{0}=\partial_{\mu} l^{\mu}$, from (30) we deduce that

$$
\delta a_{1}=\partial_{\mu}\left(f_{a b}^{c} F_{c}^{\mu \nu} A_{\nu}^{b} \eta^{a}\right)-\gamma\left(\frac{1}{2} f_{a b}^{c} F_{c}^{\mu \nu} A_{\mu}^{a} A_{\nu}^{b}\right)+(-)^{\varepsilon^{\varepsilon}} \frac{\delta^{L} \mathcal{L}_{0}}{\delta y^{i}} T_{a}^{i} \eta^{a} .
$$

Thus, for developing a consistent deformation procedure, it is necessary that the third term in the right hand-side of the last equation is $\gamma$-exact modulo $d$. This takes place if

$$
(-)^{\varepsilon_{i}} \frac{\delta^{L} \mathcal{L}_{0}}{\delta y^{i}} T_{a}^{i}=\partial_{\mu} j^{\mu}{ }_{a},
$$

for some local $j^{\mu}{ }_{a}$. The last relation is nothing but Noether's theorem expressing the appearance of the on-shell conserved currents $j^{\mu}{ }_{a}$ (on-shell means, 
as usually, on the stationary surface of field equations) deriving from the invariance of the Lagrangian action of the matter fields under the global symmetries

$$
\Delta y^{i}=T^{i}{ }_{a}\left(y^{i}, \partial_{\mu} y^{i}, \ldots, \partial_{\mu_{1}} \cdots \partial_{\mu_{k}} y^{i}\right) \xi^{a},
$$

with $\xi^{a}$ some bosonic constant parameters. In conclusion, the consistency of our deformation procedure requires in the first place that the matter theory is invariant under the rigid transformations (33), which lead to Noether's theorem (32). In the sequel we will assume that the matter theory indeed meets this requirement. The eq. (32) may be rewritten in terms of the Koszul-Tate differential as

$$
\partial_{\mu} j_{a}^{\mu}=\delta\left(-y_{i}^{*} T_{a}^{i}\right) \equiv \delta \alpha_{a},
$$

and it expresses the relationship between the rigid symmetries (33) and the homological space $H_{1}(\delta \mid d)$. Explicitly, it shows that a global symmetry (materialized in a conserved current) defines an element $\alpha_{a}$ of $H_{1}(\delta \mid d)$, i.e., an element of antighost number equal to one that is $\delta$-closed modulo $d$. A global symmetry is said to be trivial if the corresponding $\alpha_{a}$ is in a trivial class of $H_{1}(\delta \mid d)$, hence if it is $\delta$-exact modulo $d$

$$
\alpha_{a}=\delta b_{a}+\partial_{\mu} c^{\mu}{ }_{a}, \operatorname{antigh}\left(b_{a}\right)=2, \operatorname{antigh}\left(c^{\mu}{ }_{a}\right)=1 .
$$

The currents associated with a trivial global symmetry are trivial [22], and this case will be not considered here. Inserting (32) in (31), we find that

$$
a_{0}=\frac{1}{2} f_{a b}^{c} F_{c}^{\mu \nu} A_{\mu}^{a} A_{\nu}^{b}+j^{\mu}{ }_{a} A_{\mu}^{a} .
$$

So far, we have constructed the first-order deformation of the solution to the master equation in the form

$$
\begin{aligned}
& S_{1}=\int d^{D} x\left(\frac{1}{2} f_{a b}^{c} F_{c}^{\mu \nu} A_{\mu}^{a} A_{\nu}^{b}+j^{\mu}{ }_{a} A_{\mu}^{a}+.\right. \\
& \left.f^{c}{ }_{a b} A_{c}^{* \mu} A_{\mu}^{b} \eta^{a}+y_{i}^{*} T_{a}^{i} \eta^{a}-\frac{1}{2} f^{c}{ }_{a b} \eta_{c}^{*} \eta^{a} \eta^{b}\right) .
\end{aligned}
$$

The appearance of the second term in $S_{1}$ emphasizes that the first-order coupling among vector fields and matter fields is realized at the level of the deformed Lagrangian through an interaction term of the type $\mathrm{gj}^{\mu}{ }_{a} A_{\mu}^{a}$, that connects them precisely through the conserved currents associated with the global symmetries of the matter theory. 


\subsection{Higher-order deformations}

In the following we approach the higher-order consistency of the deformation.

The eq. that governs the second-order deformation, (6), demands that the antibracket

$$
\begin{aligned}
& \left(S_{1}, S_{1}\right)=s\left(\int d^{D} x \frac{1}{2} f_{a b}^{c} f_{c d e} A_{\mu}^{a} A_{\nu}^{b} A^{d \mu} A^{e \nu}\right)+ \\
& \int d^{D} x\left(2\left(f_{a b}^{c} j_{c}^{\mu}+\frac{\delta^{R} j_{b}^{\mu}}{\delta y^{i}} T_{a}^{i}\right) A_{\mu}^{b} \eta^{a}-\right. \\
& f_{b[a}^{c} f^{b}{ }_{d e]}\left(F_{c}^{\mu \nu} A_{\mu}^{a} A_{\nu}^{e} \eta^{d}+A_{c}^{* \mu} A_{\mu}^{e} \eta^{a} \eta^{d}+\frac{1}{3} \eta_{c}^{*} \eta^{a} \eta^{d} \eta^{e}\right)+ \\
& \left.y_{i}^{*}\left(\frac{\delta^{R} T_{a}^{i}}{\delta y^{j}} T_{b}^{j}-\frac{\delta^{R} T_{b}^{i}}{\delta y^{j}} T_{a}^{j}-f_{a b}^{c} T_{c}^{i}\right) \eta^{a} \eta^{b}\right)
\end{aligned}
$$

is a $s$-coboundary, or, equivalently, that its integrand is $s$-exact modulo $d$. As the last two terms in (38) cannot be $s$-exact modulo $d$, it follows that they should be made to vanish. This holds if and only if

$$
\begin{gathered}
f_{b[a}^{c} f_{d e]}^{b}=0, \\
\frac{\delta^{R} T_{a}^{i}}{\delta y^{j}} T_{b}^{j}-\frac{\delta^{R} T_{b}^{i}}{\delta y^{j}} T^{j}{ }_{a}=f_{a b}^{c} T^{i}{ }_{c} .
\end{gathered}
$$

On the one hand, the relation (39) shows that the antisymmetric constants $f_{a b}^{c}$ satisfy Jacobi's identity, and hence they stand for the structure constants of a Lie algebra. On the other hand, the solution to the eq. (40) can be expressed in the form

$$
T_{a}^{i}=T_{a k}^{i} y^{k},
$$

where $T_{a k}^{i}$ denotes a basis of generators of the linear representation of the gauge algebra under which the matter fields $y^{i}$ transform, i.e.

$$
T_{a j}^{i} T_{b k}^{j}-T_{b j}^{i} T_{a k}^{j}=f_{a b}^{c} T_{c k}^{i} .
$$

From the last component of (37), as well as from the Jacobi identity (39), it follows that the deformed gauge transformations of the vector fields form a Lie algebra with the structure constants $f_{a b}^{c}$. In the meantime, the relations (40,42) express that the deformed gauge transformations of the matter fields also generate a Lie algebra with the same structure constants. In conclusion, 
the entire deformed gauge algebra is Lie. Substituting (3940) in (38), we derive

$$
\begin{aligned}
& \left(S_{1}, S_{1}\right)=s\left(\int d^{D} x \frac{1}{2} f_{a b}^{c} f_{c d e} A_{\mu}^{a} A_{\nu}^{b} A^{d \mu} A^{e \nu}\right)+ \\
& \int d^{D} x 2\left(f_{a b}^{c} j_{c}^{\mu}+\frac{\delta^{R} j_{b}^{\mu}}{\delta y^{i}} T_{a}^{i}\right) A_{\mu}^{b} \eta^{a} .
\end{aligned}
$$

Looking at (43), we observe that two main cases arise. First, it might happen that the currents transform under the gauge version of the rigid symmetries of the matter fields according to the adjoint representation of the Lie gauge algebra, hence they satisfy the relations

$$
f_{a b}^{c} j_{c}^{\mu}+\frac{\delta^{R} j_{b}^{\mu}}{\delta y^{i}} T_{a}^{i}=0 .
$$

This further leads to $\left(S_{1}, S_{1}\right)=s\left(\int d^{D} x \frac{1}{2} f_{a b}^{c} f_{c d e} A_{\mu}^{a} A_{\nu}^{b} A^{d \mu} A^{e \nu}\right)$, such that

$$
S_{2}=-\frac{1}{4} \int d^{D} x f_{a b}^{c} f_{c d e} A_{\mu}^{a} A_{\nu}^{b} A^{d \mu} A^{e \nu}
$$

As $\left(S_{1}, S_{2}\right)=0$, we can safely take all the higher-order deformations equal to zero, $S_{3}=S_{4}=\cdots=0$, in which case the deformed solution to the master equation consistent to all orders in the coupling constant is given by

$$
\begin{aligned}
& S=\int d^{D} x\left(-\frac{1}{4} \tilde{F}_{\mu \nu}^{a} \tilde{F}_{a}^{\mu \nu}+\mathcal{L}_{0}\left(y^{i}, \partial_{\mu} y^{i}, \ldots, \partial_{\mu_{1}} \cdots \partial_{\mu_{k}} y^{i}\right)+g j_{a}^{\mu} A_{\mu}^{a}+\right. \\
& \left.A_{a}^{* \mu}\left(D_{\mu}\right)_{b}^{a} \eta^{b}+g y_{i}^{*} T_{a k}^{i} y^{k} \eta^{a}-\frac{1}{2} g f_{a b}^{c} \eta_{c}^{*} \eta^{a} \eta^{b}\right)
\end{aligned}
$$

where

$$
\begin{gathered}
\tilde{F}_{\mu \nu}^{a}=\partial_{\mu} A_{\nu}^{a}-\partial_{\nu} A_{\mu}^{a}-g f_{b c}^{a} A_{\mu}^{b} A_{\nu}^{c}, \\
\left(D_{\mu}\right)^{a}{ }_{b}=\delta^{a}{ }_{b} \partial_{\mu}+g f^{a}{ }_{b c} A_{\mu}^{c},
\end{gathered}
$$

represents the field strength of non-abelian gauge fields, respectively, the covariant derivative in the adjoint representation. From the antifield-independent piece in (46) we read that the overall Lagrangian action of the interacting gauge theory has the expression

$$
\bar{S}=\int d^{D} x\left(-\frac{1}{4} \tilde{F}_{\mu \nu}^{a} \tilde{F}_{a}^{\mu \nu}+\mathcal{L}_{0}\left(y^{i}, \partial_{\mu} y^{i}, \ldots, \partial_{\mu_{1}} \cdots \partial_{\mu_{k}} y^{i}\right)+g j^{\mu}{ }_{a} A_{\mu}^{a}\right),
$$


while from the components linear in the antifields we conclude that it is invariant under the gauge transformations

$$
\bar{\delta}_{\epsilon} A_{\mu}^{a}=\left(D_{\mu}\right)_{b}^{a} \epsilon^{b}, \bar{\delta}_{\epsilon} y^{i}=g T_{a k}^{i} y^{k} \epsilon^{a} .
$$

Thus, if the currents present in the purely matter theory transform under the gauge version of the rigid symmetries of the matter fields according to the adjoint representation of the Lie gauge algebra, then the only interacting term that couples the vector fields to the matter fields is of the type $j_{a}^{\mu} A_{\mu}^{a}$.

In the opposite case, where the currents do not satisfy (44),

$$
f_{a b}^{c} j_{c}^{\mu}+\frac{\delta^{R} j_{b}^{\mu}}{\delta y^{i}} T_{a}^{i} \neq 0,
$$

it follows that the second term from the right hand-side of (43) is nonvanishing, hence the second-order deformation involving vector fields coupled to matter fields will also be non-trivial. Moreover, it is possible to obtain other non-trivial higher-order deformations when solving the appropriate eqs., whose expressions depend on the structure of the matter theory, and hence cannot be output in the general case discussed so far. Anyway, the complete deformed solution to the master equation starts like

$$
S^{\prime}=S+g^{2} S_{2}+\cdots,
$$

such that the Lagrangian action of the interacting theory is of the type

$$
\bar{S}^{\prime}=\bar{S}+\mathcal{O}\left(g^{2}\right)
$$

In this situation, the gauge transformations of the fields involved with the interacting theory are also given by (50).

The deformation treatment developed so far can be synthesized in three general results as follows. First, the interaction terms involving only the vector fields generate the Lagrangian action of Yang-Mills theory, and the first-order couplings between the vector fields and matter fields is of the type $j^{\mu}{ }_{a} A_{\mu}^{a}$. Second, the gauge transformations of the vector fields are modified with respect to the initial ones, while those of the matter fields can be obtained by simply gauging the original rigid ones. Third, the overall deformed gauge algebra is a Lie algebra. Finally, a word of caution. Once the deformations related to a given matter theory are computed, special attention should be paid to the elimination of non-locality, as well as of triviality of the resulting deformations. This completes our general procedure. 


\section{Applications}

Next, we consider two examples of matter theories - real scalar fields and Dirac fields - and compute their consistent interactions with a set of vector fields in the light of the analysis performed in the previous section.

\subsection{Vector fields coupled to scalar fields}

First, we analyse the consistent interactions that can be introduced among a set of real scalar fields and a collection of vector fields. In this case the "free" Lagrangian action (8) is given by

$$
\tilde{S}_{0}^{L}\left[A_{\mu}^{a}, \varphi^{A}\right]=\int d^{4} x\left(-\frac{1}{4} F_{\mu \nu}^{a} F_{a}^{\mu \nu}+\frac{1}{2} K_{A B}\left(\partial_{\mu} \varphi^{A}\right)\left(\partial^{\mu} \varphi^{B}\right)-V\left(\varphi^{A}\right)\right)
$$

where $K_{A B}$ is an invertible symmetric constant matrix. The "free" KoszulTate differential and the exterior derivative along the gauge orbits act on the generators associated with the matter sector $y^{i}=\left(\varphi^{A}\right), y_{i}^{*}=\left(\varphi_{A}^{*}\right)$ like

$$
\begin{gathered}
\delta \varphi^{A}=0, \gamma \varphi^{A}=0, \\
\delta \varphi_{A}^{*}=K_{A B} \partial_{\mu} \partial^{\mu} \varphi^{B}+\frac{\partial V}{\partial \varphi^{A}}, \gamma \varphi_{A}^{*}=0,
\end{gathered}
$$

while on the purely gauge ones $A_{\mu}^{a}, \eta^{a}, A_{a}^{* \mu}$ and $\eta_{a}^{*}$ like in the first and third relations from each of the eqs. (15 18). Multiplying (56) by $-T_{a C}^{A} \varphi^{C}$, we arrive at the eq.

$$
\begin{aligned}
& \delta\left(-\varphi_{A}^{*} T_{a C}^{A} \varphi^{C}\right)=\partial_{\mu}\left(-K_{A B} T_{a C}^{A}\left(\partial^{\mu} \varphi^{B}\right) \varphi^{C}\right)+ \\
& K_{A B} T_{a C}^{A}\left(\partial^{\mu} \varphi^{B}\right)\left(\partial_{\mu} \varphi^{C}\right)-\frac{\partial V}{\partial \varphi^{A}} T_{a C}^{A} \varphi^{C} .
\end{aligned}
$$

In order to have a non-trivial local homology for $\delta$ at antighost number one, the right hand-side of (57) should reduce to a total derivative. This takes place if and only if

$$
\begin{aligned}
& \frac{\partial V}{\partial \varphi^{A}} T_{a C}^{A} \varphi^{C}=0, \\
& \tilde{T}_{a B C}=-\tilde{T}_{a C B},
\end{aligned}
$$


with $\tilde{T}_{a B C} \equiv K_{A B} T_{a C}^{A}$. The equations (58-59) ensure the invariance of the action of the scalar theory under the rigid transformations $\Delta \varphi^{A}=T_{a C}^{A} \varphi^{C} \xi^{a}$, such that we obtain the conservation of the currents

$$
j^{\mu}{ }_{a}=-K_{A B} T_{a C}^{A}\left(\partial^{\mu} \varphi^{B}\right) \varphi^{C} .
$$

Then, with the help of (37) and (60), the first-order deformation of the solution to the master equation reads

$$
\begin{aligned}
& S_{1}=\int d^{4} x\left(\frac{1}{2} f_{a b}^{c} F_{c}^{\mu \nu} A_{\mu}^{a} A_{\nu}^{b}-K_{A B} T_{a C}^{A}\left(\partial^{\mu} \varphi^{B}\right) \varphi^{C} A_{\mu}^{a}+.\right. \\
& \left.f_{a b}^{c} A_{c}^{* \mu} A_{\mu}^{b} \eta^{a}+\varphi_{A}^{*} T_{a C}^{A} \varphi^{C} \eta^{a}-\frac{1}{2} f_{a b}^{c} \eta_{c}^{*} \eta^{a} \eta^{b}\right) .
\end{aligned}
$$

Now, we investigate the second-order deformation. In view of this, we observe that the second term in the right hand-side of (43) adapted to the model under study is given by

$$
2\left(f_{a b}^{c} j_{c}^{\mu}+\frac{\delta^{R} j_{b}^{\mu}}{\delta \varphi^{A}} T_{a C}^{A} \varphi^{C}\right) A_{\mu}^{b} \eta^{a}=s\left(K_{A B} T_{a D}^{A} T_{b C}^{D} \varphi^{B} \varphi^{C} A_{\mu}^{a} A^{b \mu}\right),
$$

such that

$$
\begin{aligned}
& S_{2}=\int d^{D} x\left(-\frac{1}{4} f_{a b}^{c} f_{c d e} A_{\mu}^{a} A_{\nu}^{b} A^{d \mu} A^{e \nu}+\right. \\
& \left.\frac{1}{2} K_{A D} T_{a B}^{A} T_{b C}^{D} \varphi^{B} \varphi^{C} A_{\mu}^{a} A^{b \mu}\right) .
\end{aligned}
$$

This case corresponds to the second situation described in the final part of Sec. 3. If we examine the third-order deformation (see eq. (77)), we notice that $\left(S_{1}, S_{2}\right)=0$, hence we can safely take $S_{3}=0$. The higher-order eqs. are then satisfied with the choice $S_{4}=S_{5}=\cdots=0$. Putting together the above results, we infer that

$$
\begin{aligned}
& S^{\prime}=\int d^{4} x\left(-\frac{1}{4} \tilde{F}_{\mu \nu}^{a} \tilde{F}_{a}^{\mu \nu}+\frac{1}{2} K_{A B}\left(D_{\mu C}^{A} \varphi^{C}\right)\left(D_{D}^{\mu B} \varphi^{D}\right)-V\left(\varphi^{A}\right)+\right. \\
& \left.g \varphi_{A}^{*} T_{a C}^{A} \varphi^{C} \eta^{a}+A_{a}^{* \mu}\left(D_{\mu}\right)_{b}^{a} \eta^{b}-\frac{1}{2} g f_{a b}^{c} \eta_{c}^{*} \eta^{a} \eta^{b}\right)
\end{aligned}
$$

represents the full consistent solution to the master equation of our deformed problem, where the covariant derivative that acts on the matter fields is defined through

$$
D_{\mu C}^{A}=\delta_{C}^{A} \partial_{\mu}+g T_{a C}^{A} A_{\mu}^{a} .
$$


The antifield-independent piece in (64)

$$
\bar{S}^{\prime}=\int d^{4} x\left(-\frac{1}{4} \tilde{F}_{\mu \nu}^{a} \tilde{F}_{a}^{\mu \nu}+\frac{1}{2} K_{A B}\left(D_{\mu C}^{A} \varphi^{C}\right)\left(D_{D}^{\mu B} \varphi^{D}\right)-V\left(\varphi^{A}\right)\right),
$$

describes nothing but the Lagrangian interaction between a set of real scalar fields and a collection of vector fields, while the terms linear in the antifields of the matter fields give the gauge transformations

$$
\bar{\delta}_{\epsilon} \varphi^{A}=g T_{a C}^{A} \varphi^{C} \epsilon^{a},
$$

those associated with the vector fields being as in the former relations from (50). If in (66 67) we make the transformation $T_{a C}^{A} \rightarrow i T_{a C}^{A}$, we arrive at the non-abelian analogue of scalar electrodynamics.

\subsection{Vector fields coupled to Dirac fields}

Finally, we examine the consistent couplings between a set of vector fields and a collection of massive Dirac fields. In view of this, we start from the Lagrangian action

$$
\tilde{S}_{0}^{L}\left[A_{\mu}^{a}, \psi_{A}^{\alpha}, \bar{\psi}_{\alpha}^{A}\right]=\int d^{4} x\left(-\frac{1}{4} F_{\mu \nu}^{a} F_{a}^{\mu \nu}+\bar{\psi}_{\alpha}^{A}\left(i\left(\gamma^{\mu}\right)_{\beta}^{\alpha} \partial_{\mu}-m \delta_{\beta}^{\alpha}\right) \psi_{A}^{\beta}\right),
$$

where $\psi_{A}^{\alpha}$ and $\bar{\psi}_{\alpha}^{A}$ denote the fermionic spinor components of the Dirac fields $\psi_{A}$ and $\bar{\psi}^{A}$. The actions of $\delta$ and $\gamma$ on the matter generators from the free BRST complex are expressed by

$$
\begin{gathered}
\delta \psi_{A}^{\alpha}=0, \delta \bar{\psi}_{\alpha}^{A}=0, \\
\delta \psi_{\alpha}^{* A}=-\left(i\left(\gamma^{\mu}\right)_{\alpha}^{\beta} \partial_{\mu}+m \delta_{\alpha}^{\beta}\right) \bar{\psi}_{\beta}^{A}, \\
\delta \bar{\psi}_{A}^{* \alpha}=-\left(i\left(\gamma^{\mu}\right)_{\beta}^{\alpha} \partial_{\mu}-m \delta_{\beta}^{\alpha}\right) \psi_{A}^{\beta}, \\
\gamma \psi_{A}^{\alpha}=0, \gamma \bar{\psi}_{\alpha}^{A}=0, \gamma \psi_{\alpha}^{* A}=0, \gamma \bar{\psi}_{A}^{* \alpha}=0,
\end{gathered}
$$

where the antighost number one antifields $\psi_{\alpha}^{* A}$ and $\bar{\psi}_{A}^{* \alpha}$ are bosonic. Multiplying (70) and (71) from the right by $T_{a A}^{C} \psi_{C}^{\alpha}$, respectively, $-T_{a C}^{A} \bar{\psi}_{\alpha}^{C}$, and subtracting the resulting relations, we deduce

$$
\delta\left(\psi_{\alpha}^{* A} T_{a A}^{C} \psi_{C}^{\alpha}-\bar{\psi}_{A}^{* \alpha} T_{a C}^{A} \bar{\psi}_{\alpha}^{C}\right)=\partial_{\mu}\left(i \bar{\psi}_{\alpha}^{A}\left(\gamma^{\mu}\right)_{\beta}^{\alpha} T_{a A}^{C} \psi_{C}^{\beta}\right),
$$


which underlines the conservation of the currents

$$
j^{\mu}{ }_{a}=i \bar{\psi}_{\alpha}^{A}\left(\gamma^{\mu}\right)_{\beta}^{\alpha} T_{a A}^{C} \psi_{C}^{\beta},
$$

resulting from the global invariances $\Delta \psi_{A}^{\alpha}=-T_{a A}^{C} \psi_{C}^{\alpha} \xi^{a}$ and $\Delta \bar{\psi}_{\alpha}^{A}=T_{a C}^{A} \bar{\psi}_{\alpha}^{C} \xi^{a}$ of the massive Dirac theory. Acting like before, the first-order deformed solution to the master equation is given by

$$
\begin{aligned}
& S_{1}=\int d^{4} x\left(\frac{1}{2} f_{a b}^{c} F_{c}^{\mu \nu} A_{\mu}^{a} A_{\nu}^{b}+i \bar{\psi}_{\alpha}^{A}\left(\gamma^{\mu}\right)_{\beta}^{\alpha} T_{a A}^{C} \psi_{C}^{\beta} A_{\mu}^{a}+\right. \\
& \left.f_{a b}^{c} A_{c}^{* \mu} A_{\mu}^{b} \eta^{a}-\left(\psi_{\alpha}^{* A} T_{a A}^{C} \psi_{C}^{\alpha}-\bar{\psi}_{A}^{* \alpha} T_{a C}^{A} \bar{\psi}_{\alpha}^{C}\right) \eta^{a}-\frac{1}{2} f_{a b}^{c} \eta_{c}^{*} \eta^{a} \eta^{b}\right) .
\end{aligned}
$$

With this solution at hand, we observe that the second term in the right hand-side of (43) adapted to the model under study vanishes

$$
f_{a b}^{c} j_{c}^{\mu}+\frac{\delta^{R} j_{b}^{\mu}}{\delta \bar{\psi}_{\alpha}^{A}} T_{a B}^{A} \bar{\psi}_{\alpha}^{B}-\frac{\delta^{R} j_{b}^{\mu}}{\delta \psi_{A}^{\alpha}} T_{a A}^{B} \psi_{B}^{\alpha}=0,
$$

so $S_{2}$ is given by (45). In this light, we conclude that the present model satisfies the conditions described in the first situation discussed in the final part of Sec. 3. Consequently, we can set $S_{3}=0$, and, moreover, $S_{4}=S_{5}=$ $\cdots=0$. The complete deformed solution to the master equation that is consistent to all orders in the deformation parameter is

$$
\begin{aligned}
& S=\int d^{4} x\left(-\frac{1}{4} \tilde{F}_{\mu \nu}^{a} \tilde{F}_{a}^{\mu \nu}+\bar{\psi}_{\alpha}^{A}\left(i\left(\gamma^{\mu}\right)^{\alpha}{ }_{\beta} D_{\mu A}^{C}-m \delta_{\beta}^{\alpha} \delta_{A}^{C}\right) \psi_{C}^{\beta}+\right. \\
& A_{a}^{* \mu}\left(D_{\mu}\right)^{a}{ }_{b} \eta^{b}-g\left(\psi_{\alpha}^{* A} T_{a A}^{C} \psi_{C}^{\alpha}-\bar{\psi}_{A}^{* \alpha} T_{a C}^{A} \bar{\psi}_{\alpha}^{C}\right) \eta^{a}- \\
& \left.\frac{1}{2} g f_{a b}^{c} \eta_{c}^{*} \eta^{a} \eta^{b}\right) .
\end{aligned}
$$

Its antifield-independent piece

$$
\bar{S}=\int d^{4} x\left(-\frac{1}{4} \tilde{F}_{\mu \nu}^{a} \tilde{F}_{a}^{\mu \nu}+\bar{\psi}_{\alpha}^{A}\left(i\left(\gamma^{\mu}\right)_{\beta}^{\alpha} D_{\mu A}^{C}-m \delta_{\beta}^{\alpha} \delta_{A}^{C}\right) \psi_{C}^{\beta}\right),
$$

reveals the Lagrangian interaction between a set of vector fields and a collection of massive Dirac fields, while from the terms linear in the antifields of the matter fields we notice the gauge transformation of the Dirac fields are

$$
\delta_{\epsilon} \psi_{A}^{\alpha}=-g T_{a A}^{C} \psi_{C}^{\alpha} \epsilon^{a}, \delta_{\epsilon} \bar{\psi}_{\alpha}^{A}=g T_{a C}^{A} \bar{\psi}_{\alpha}^{C} \epsilon^{a},
$$

those associated with the vector fields being as in the former relations from (50). Similar to the scalar case, if in (78 79) we make the transformation $T_{a C}^{A} \rightarrow i T_{a C}^{A}$ and consider an $S U(3)$ gauge algebra, we arrive at quantum chromodynamics. 


\section{Conclusion}

In conclusion, in this paper we have investigated the consistent interactions that can be introduced between a set of vector fields and a system of matter fields by using some cohomological techniques. In this context, we have shown that the first-order interaction contains two terms. The first one describes an interaction among the vector fields (the cubic vertex of Yang-Mills theory). The second term appears only if the matter theory displays as many global symmetries as there are vector fields, and is of the form $j_{a}^{\mu} A_{\mu}^{a}$, where $j^{\mu}{ }_{a}$ are the conserved currents of the matter theory corresponding to the above mentioned global symmetries. The consistency of the first-order deformation requires that the deformed gauge algebra is Lie, and, in the meantime, emphasizes that the second-order deformation contains the quartic vertex of pure Yang-Mills theory. If the conserved currents $j^{\mu}{ }_{a}$ transform under the gauge version of the rigid symmetries of the matter fields according to the adjoint representation of the Lie gauge algebra, then all the other deformations involving the matter fields, of order two and higher, vanish. In the opposite case, at least the second-order deformation implying matter fields is non-vanishing, but in principle there might be other non-trivial terms. The general procedure has been applied to the study of the interactions between a set of vector fields and a collection of real scalar fields, respectively, a set of Dirac fields.

\section{Acknowledgment}

This work has been supported by a Romanian National Council for Academic Scientific Research (CNCSIS) grant.

\section{References}

[1] I. A. Batalin, G. A. Vilkovisky, Phys. Lett. B102 (1981) 27; Phys. Rev. D28 (1983) 2567; J. Math. Phys. 26 (1985) 172; M. Henneaux, Nucl. Phys. B (Proc. Suppl.) 18A (1990) 47

[2] M. Henneaux, C. Teitelboim, Quantization of Gauge Systems, Princeton Univ. Press, Princeton 1992 
[3] G. Barnich, M. Henneaux, Phys. Lett. B311 (1993) 123

[4] J. Stasheff, Deformation theory and the Batalin-Vilkovisky master equation, q-alg/9702012

[5] J. Stasheff, The (secret?) homological algebra of the Batalin-Vilkovisky approach, hep-th/9712157

[6] J. A. Garcia, B. Knaepen, Phys. Lett. B441 (1998) 198

[7] G. Barnich, F. Brandt, M. Henneaux, Commun. Math. Phys. 174 (1995) 93; G. Barnich, M. Henneaux, R. Tatar, Int. J. Mod. Phys. D3 (1994) 139

[8] M. Henneaux, B. Knaepen, C. Schomblond, Lett. Math. Phys. 42 (1997) 337; C. Bizdadea, L. Saliu, S. O. Saliu, Phys. Scripta 61 (2000) 307

[9] M. Henneaux, Phys. Lett. B368 (1996) 83; C. Bizdadea, M. G. Mocioacă, S. O. Saliu, Phys. Lett. B459 (1999) 145

[10] M. Henneaux, B. Knaepen, Phys. Rev. D56, 6076 (1997)

[11] X. Bekaert, M. Henneaux, Int. J. Theor. Phys. 38, 1161 (1999)

[12] X. Bekaert, M. Henneaux, A. Sevrin, Phys. Lett. B468, 228 (1999)

[13] X. Bekaert, M. Henneaux, A. Sevrin, Nucl. Phys. B (Proc. Suppl.) 88 (2000) 27

[14] K. -I. Izawa, Progr. Theor. Phys. 103 (2000) 225

[15] G. Barnich, F. Brandt, M. Henneaux, Nucl. Phys. B455 (1995) 357; R. Wald, Phys. Rev. D33 (1986) 3613

[16] F. Brandt, Ann. Phys. (N.Y.) 259 (1997) 253; E. Cremmer, B. Julia, J. Scherk, Phys. Lett. B76 (1978) 409

[17] C. Bizdadea, hep-th/0003199

[18] C. Bizdadea, E. M. Cioroianu, S. O. Saliu, Class. Quantum Grav. 17 (2000) 2007

[19] C. Bizdadea, L. Saliu, S. O. Saliu, Int. J. Mod. Phys. A15 (2000) 893 
[20] C. Bizdadea, S. O. Saliu, Phys. Scripta 62 (2000) 261

[21] C. Bizdadea, E. M. Cioroianu, I. Negru, S. O. Saliu, to appear in Ann. Phys. (Leipzig)

[22] G. Barnich, F. Brandt, M. Henneaux, Commun. Math. Phys. 174 (1995) 57; G. Barnich, F. Brandt, M. Henneaux, Phys. Rep. 338 (2000) 439 